\title{
COVID-19 May Cause Severe Illness in Diabetic Patients
}

\author{
Abu Saim Mohammad Saikat $1, *$ \\ ${ }^{1}$ Department of Biochemistry and Molecular Biology, Bangabandhu Sheikh Mujibur Rahman Science \\ and Technology University, Gopalganj, Bangladesh \\ "Corrresponding author: asmsaikat.bmb@gmail.com
}

Received: May 24, 2020; revised: May 31, 2020; accepted: June 6, 2020.

\begin{abstract}
COVID-19 pandemic has become the top public health concern worldwide that represents a threat to the life of billions of people globally. Common causes of death of COVID-19 infected individuals are multi-organ failure, pneumonia, and acute respiratory distress syndrome, out of which pneumonia is the most common. COVID-19 patients with diabetes are at high risk for medical complications, which may lead to severe complications, even death.
\end{abstract}

Keywords: COVID-19, diabetes, coronavirus, hyperglycemia, ACE inhibitor

Coronavirus Disease 2019 (COVID-19), a highly fatal disease named by the World Health Organization (WHO), is a kind of viral pneumonia caused by the Severe Acute Respiratory Syndrome Coronavirus 2 (SARS-CoV-2). Previously known as the 2019 novel Coronavirus (2019nCoV), it has started spreading since December 2019 from the center of this outbreak, Wuhan city, the capital of Hubei province in China (WHO, 2020b; Kwong et al., 2020). WHO has declared the novel coronavirus (COVID-19) outbreak a global pandemic on March 11, 2020. Globally, as of the 31 May 2020, there have been 5,939,234 confirmed cases of COVID-19, including 367,255 deaths, reported to WHO (2020a). There are 47,153 COVID19 confirmed cases in Bangladesh, including 650 deaths, and 9,781 recovered against the total tests of 308,940 as of 31 May 2020 (IEDCR, 2020). Diabetes patients infected with COVID-19 are associated with a two-fold increase in severity as well as mortality, as compared to non-diabetics. Further studies on the therapeutic implications and the pathogenic mechanisms are needed to be done (Kumar et al., 2020).

The presence of diabetes in COVID-19 patients increases the risk for medical complications e.g., death (Hill et al., 2020; Zhou and Tan, 2020). During the COVID-19 infections, 
the patients may stress out about the importance of glycemic control. They may adversely affect the immune response and pulmonary function due to hyperglycemia (Klonoff and Umpierrez, 2020). There are many reasons why COVID-19 patients experience inadequate glycemic control or at a higher risk due to diabetes than in the pre-COVID-19 state. Additionally, drugs, including Angiotensin II Receptor Blockers and ACE inhibitors, are used to treat diabetic patients. In contrast, the COVID-19 virus uses ACE -2 receptor for binding to different tissues e.g., kidney, endothelium, lung, etc. resulting in increased complications of diabetic patients infected with COVID-19 (Ahirwar et al., 2020).

However, there are some important rules to follow for the management of diabetic patients, including regular monitoring of blood sugar level, body temperature, ketone bodies in urine (if a patient is on insulin therapy), and regular physical exercises. The diabetic patients should keep sugar candy and biscuit with them due to avoiding hypoglycemia (will take whenever they have signs and symptoms). Vaccination for diabetic patients against influenza and pneumonia could be helpful. An appropriate diet is necessary for a diabetic individual in terms of vitamins, proteins, and minerals. Furthermore, blood glucose management of diabetic patients infected with COVID-19 should be better valued and optimized through professional advice, providing a diabetic diet or personalized diet to the patients, providing indoor exercise instructions. The anxiety levels of inpatients should be evaluated, as the anxiety led by COVID-19 induces hyperglycemia (Miazgowski et al., 2018).

Frontline doctors treating patients could experience a patient healthy in the morning and needing a ventilator in the afternoon. To address this problem, primary-care teams of the hospitals could try something new, including calling patients on a regular interval such as on days 2, 5, and 8 after they have diagnosed with COVID-19 and instruct them to self-isolate at home. COVID-19 pandemic has created mass panic globally due to false information and rumors as wells as fake news spreading in social media. This mass panic could be mitigated by creating awareness among Bangladeshi people by prominent researchers, religious scholars e.g., imam of mosques, authentic community leaders, and public figures (Hossain et al., 2020).

\section{Acknowledgments}

I would like to thank Lutful Kabir, Assistant Professor, Department of Biochemistry and Molecular Biology, Bangabandhu Sheikh Mujibur Rahman Science and Technology University, Gopalganj, Bangladesh, for reviewing the manuscript.

\section{Declarations}

Funding: There was no funding received for this commentary. Conflict of interest: The author declares no conflict of interest. Ethical approval: Not applicable. 


\section{References}

Ahirwar AK, Asia P, Sakarde A, Kaim K (2020). "COVID-19 outbreakDiabetes aspect and perspective." Current Medicine Research and Practice. doi:https://doi.org/10.1016/j.cmrp.2020.05.005.

Hill MA, Mantzoros C, Sowers JR (2020). "Commentary: COVID-19 in patients with diabetes." Metabolism. doi:https://doi.org/10.1016/j.metabol.2020.154217.

Hossain MS, Ferdous S, Siddiqee MH (2020). "Mass panic during Covid-19 outbreak-A perspective from Bangladesh as a high-risk country." Journal of Biomedical Analytics, 3(2), 1-3. doi:https://doi.org/10.30577/jba.v3i2.40.

IEDCR (2020). "Institute of Epidemiology Disease Control and Research." Accessed June 1, 2020, URL https://www.iedcr.gov.bd/.

Klonoff DC, Umpierrez GE (2020). "COVID-19 in patients with diabetes: risk factors that increase morbidity." Metabolism-Clinical and Experimental. doi:https://doi.org/10.1016/j.metabol.2020.154224.

Kumar A, Arora A, Sharma P, Anikhindi SA, Bansal N, Singla V, Khare S, Srivastava A (2020). "Is diabetes mellitus associated with mortality and severity of COVID19? A meta-analysis." Diabetes \& Metabolic Syndrome: Clinical Research \& Reviews. doi:https://doi.org/10.1016/j.dsx.2020.04.044.

Kwong KCNK, Mehta PR, Shukla G, Mehta AR (2020). "COVID-19, SARS and MERS: A neurological perspective." Journal of Clinical Neuroscience. doi:https://doi.org/10.1016/j.jocn.2020.04.124.

Miazgowski T, Bikowska M, Ogonowski J, Taszarek A (2018). "The Impact of Health Locus of Control and Anxiety on Self-Monitored Blood Glucose Concentration in Women with Gestational Diabetes Mellitus." Journal of Women's Health, 27(2), 209-215. doi:https://doi.org/10.1089/jwh.2017.6366.

WHO (2020a). "WHO Coronavirus Disease (COVID-19) Dashboard n.d." Accessed June 1, 2020, URL https://bit.ly/2XFrMH9.

WHO (2020b). "WHO Director-General's remarks at the media briefing on 2019-nCoV on 11 February 2020." Accessed May 24, 2020, URL https://www.who.int/dg/speeches/detail/ who-director-general-s-remarks-at-the-media-briefing-on-2019-ncov-on-11-february-2020.

Zhou J, Tan J (2020). "Diabetes patients with COVID-19 need better blood glucose management in Wuhan, China." Metabolism-Clinical and Experimental, 107. doi:https://doi.org/10.1016/j.metabol.2020.154216. 